\title{
Validity of the scoring system for traumatic liver injury: a generalized estimating equation analysis
}

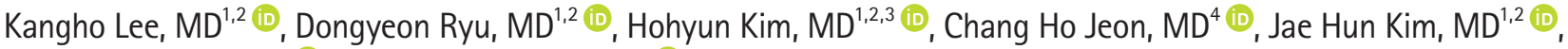 \\ Chan Yong Park, MD ${ }^{5}$ (i) , Seok Ran Yeom, MD $2,3,6$ (iD) \\ ${ }^{1}$ Department of Trauma and Surgical Critical Care, Pusan National University Hospital, Busan, Korea \\ ${ }^{2}$ Biomedical Research Institute, Pusan National University Hospital, Busan, Korea \\ ${ }^{3}$ Department of Emergency Medicine, Pusan National University School of Medicine, Yangsan, Korea \\ ${ }^{4}$ Department of Diagnostic Radiology, The Catholic University of Korea Eunpyeong St. Mary's Hospital, Seoul, Korea \\ ${ }^{5}$ Department of Trauma Surgery, Seoul National University Hospital, Seoul, Korea \\ ${ }^{6}$ Department of Emergency Medicine, Pusan National University Hospital, Busan, Korea
}

Received: January 15, 2021

Revised: February 17, 2021

Accepted: March 11, 2021

Correspondence to

Hohyun Kim, MD

Department of Trauma and Surgical

Critical Care, Pusan National University

Hospital, 179 Gudeok-ro, Seo-gu,

Busan 49241, Korea

Tel: +82-51-240-7369

E-mail: raumagskhh@pusan.ac.kr
Purpose: The scoring system for traumatic liver injury (SSTLI) was developed in 2015 to predict mortality in patients with polytraumatic liver injury. This study aimed to validate the SSTLI as a prognostic factor in patients with polytrauma and liver injury through a generalized estimating equation analysis.

Methods: The medical records of 521 patients with traumatic liver injury from January 2015 to December 2019 were reviewed. The primary outcome variable was in-hospital mortality. All the risk factors were analyzed using multivariate logistic regression analysis. The SSTLI has five clinical measures (age, Injury Severity Score, serum total bilirubin level, prothrombin time, and creatinine level) chosen based on their predictive power. Each measure is scored as 0-1 (age and Injury Severity Score) or 0-3 (serum total bilirubin level, prothrombin time, and creatinine level). The SSTLI score corresponds to the total points for each item (0-11 points).

Results: The areas under the curve of the SSTLI to predict mortality on post-traumatic days $0,1,3$, and 5 were $0.736,0.783,0.830$, and 0.824 , respectively. A very good to excellent positive correlation was observed between the probability of mortality and the SSTLI score $(\gamma=0.997, \mathrm{P}<0.001)$. A value of 5 points was used as the threshold to distinguish low-risk $(<5)$ from high-risk $(\geq 5)$ patients. Multivariate analysis using the generalized estimating equation in the logistic regression model indicated that the SSTLI score was an independent predictor of mortality (odds ratio, 1.027; 95\% confidence interval, 1.018-1.036; $\mathrm{P}<0.001$ ).

Conclusions: The SSTLI was verified to predict mortality in patients with polytrauma and liver injury. A score of $\geq 5$ on the SSTLI indicated a high-risk of post-traumatic mortality.

Keywords: Wounds and injuries; Liver; Trauma severity indices; Prognosis 


\section{INTRODUCTION}

The abdominal cavity is the third most common site affected by trauma [1]. The liver is the most frequently injured organ in abdominal trauma despite being relatively shielded by the ribs [25], and liver trauma is the leading cause of death in major abdominal trauma $[6,7]$. Therefore, one of the most important roles of trauma centers is the successful treatment of liver injuries. Previously, patients with liver trauma underwent surgical treatment with techniques including packing, hepatorrhaphy, vessel ligation, and hepatic resection [8]. Emergent operative management $(\mathrm{OM})$ for liver injuries in hemodynamically unstable patients is essential [7,9]. However, nonoperative management (NOM), including watchful waiting and/or arterial embolization, is the preferred treatment modality in hemodynamically stable patients $[7,10]$.

There are various scoring systems to establish the physiological severity of injuries and the prognoses of patients who have sustained trauma. The Acute Physiology and Chronic Health Evaluation Score [11], Injury Severity Score (ISS) [12], Revised Trauma Score [13], and Trauma and Injury Severity Score [14] are used to determine the severity and prognosis of injuries. However, these scoring systems are not specific to liver trauma; they are specific to various other types of injuries [15].

Given the lack of a liver-specific scoring system despite the importance of liver trauma treatment, we published a study that examined prognostic factors in patients with polytrauma and liver injury and developed a scoring system for traumatic liver injury (SSTLI) to predict mortality in 2015 [15]. This study aimed to validate the SSTLI as a prognostic factor in patients with polytrauma and liver injury.

\section{METHODS}

This study was approved by the Institutional Review Board of the Pusan National University Hospital, Busan (No. H-2012-022-098).

\section{Components of the SSTLI}

We published a study on the SSTLI in 2015 using patients' laboratory values and initial history data [15]. As shown in Table 1, the SSTLI uses five clinical measures (age, ISS, serum total bilirubin level, prothrombin time, and creatinine level), and each measure was scored from 0 to 1 (age and ISS) or from 0 to 3 (serum total bilirubin level, prothrombin time, and creatinine level). The SSTLI score corresponds to the sum of points for each item (0-11 points).
Table 1. The scoring system for traumatic liver injury

\begin{tabular}{lcccc}
\hline \multirow{2}{*}{ Measure } & \multicolumn{5}{c}{ Point } \\
\cline { 2 - 5 } & 0 & 1 & 2 & 3 \\
\hline Age $(\mathrm{yr})$ & $<60$ & $\geq 60$ & - & - \\
Injury Severity Score & $<25$ & $\geq 25$ & - & - \\
Serum total bilirubin (mg/dL) & $<1$ & $1-2$ & $2-3$ & $>3$ \\
Prothrombin time (INR) & $<1$ & $1-1.7$ & $1.7-2.3$ & $>2.3$ \\
Serum creatinine (mg/dL) & $<1$ & $1-2$ & $2-3$ & $>3$
\end{tabular}

INR, international normalized ratio.

\section{Study population}

Patients with polytrauma and liver injury were included in this study because liver trauma is almost always accompanied by other organ injuries. In total, 564 patients with polytrauma and liver injury admitted to the emergency room at Pusan National University Hospital, Busan between January 2015 and December 2019 were considered for this study. Patients who died within 24 hours or who were discharged or transferred within 7 days were excluded because they were judged to be unsuitable for determining patients' prognosis after successful resuscitation. With the additional exclusion of those with inadequate medical records, 43 patients were excluded. Therefore, 521 patients were enrolled in this study. If a patient was hemodynamically stable after initial resuscitation, had a normal mental status, and had no signs of peritoneal irritation upon arrival at the emergency room, the patient underwent NOM. According to this protocol, 446 patients underwent $\mathrm{NOM}$ and 75 patients underwent $\mathrm{OM}$.

We performed a retrospective chart review of 521 patients with polytrauma and liver injury. We collected data on age, sex, and laboratory values (levels of total bilirubin, prothrombin time, and creatinine) from electronic medical records.

\section{Outcome measures}

The primary outcome variable was in-hospital mortality. Clinical variables were analyzed to identify factors predicting mortality after hospital management.

\section{Statistical analysis}

The Mann-Whitney U-test and the Wilcoxon rank-sum test were used to compare the mean values of the continuous variables and ordinal data, respectively. The chi-square test and Fisher exact test were used to compare the frequencies of categorical variables between groups. We modelled repeated measurements of all variables related to the SSTLI over time using a generalized estimating equation (GEE) extension of a logistic regression model [16]. We used the receiver operating characteris- 
tic curve and area under the curve (AUC) to evaluate prognostic factors predicting death. All risk factors were analyzed using multivariate logistic regression analysis. The SSTLI was created based on the predictive power of each factor. A P-value of $\leq 0.05$ was considered to indicate statistical significance. All statistical analyses were performed using IBM SPSS ver. 20.0 (IBM Corp., Armonk, NY, USA) and Stata ver. 14.2 (Stata Corp., College Station, TX, USA).

\section{RESULTS}

\section{Clinical characteristics of patients with traumatic liver} injury

The clinical characteristics of patients (382 male patients [73.3\%] and 139 female patients [26.7\%]; median age, 50 years; inter- quartile range [IQR], 33-61 years) are shown in Table 2. The average ISS was 22 (IQR, 4-50). In total, 446 patients (85.6\%) underwent NOM, 75 patients (14.4\%) underwent OM, and 165 patients (31.7\%) underwent angiography (Fig. 1).

Of the 521 patients, 30 patients (5.8\%) died in the hospital. There was no significant difference between survivors and nonsurvivors with regard to sex. The median age, median ISS, and percentage of $\mathrm{OM}$ were higher in nonsurvivors than in survivors $(\mathrm{P}=0.037, \mathrm{P}<0.001$, and $\mathrm{P}=0.002$, respectively). The serum total bilirubin level (after post-traumatic day [PTD] 3), prothrombin time, and creatinine level were higher in nonsurvivors than in survivors. The AUC values for age and ISS showed a significant increase relative to the null curve (AUC $=0.613, \mathrm{P}=0.037$ and $\mathrm{AUC}=0.740, \mathrm{P}<0.001$, respectively) (Fig. $\mathrm{S} 1$ ).

Table 2. Clinical characteristics of patients with traumatic liver injury

\begin{tabular}{|c|c|c|c|c|}
\hline Characteristics & Survivor group $(n=491)$ & Nonsurvivor group $(n=30)$ & Total $(n=521)$ & P-value \\
\hline Age (yr) & $49(33-60)$ & $56(47-67)$ & $50(33-61)$ & 0.037 \\
\hline Age group (yr) & & & & 0.048 \\
\hline$<60$ & $360(73.3)$ & $17(56.7)$ & $277(72.4)$ & \\
\hline$\geq 60$ & $131(26.7)$ & $13(43.3)$ & $144(27.6)$ & \\
\hline Sex (male:female) & $360: 131$ & $22: 8$ & $382: 139$ & 0.999 \\
\hline ISS & $22(17-29)$ & $29(25-41)$ & $22(4-50)$ & $<0.001$ \\
\hline ISS group & & & & $<0.001$ \\
\hline$<25$ & $284(57.8)$ & $7(23.3)$ & $281(55.9)$ & \\
\hline$\geq 25$ & $207(42.2)$ & $23(76.7)$ & $230(44.1)$ & \\
\hline Operation & & & & 0.002 \\
\hline NOM & $426(86.8)$ & $20(66.7)$ & $446(85.6)$ & \\
\hline $\mathrm{OM}$ & $65(13.2)$ & $10(33.3)$ & $75(14.4)$ & \\
\hline Angiography & $153(31.2)$ & $12(40.0)$ & $165(31.7)$ & 0.312 \\
\hline \multicolumn{5}{|c|}{ Total bilirubin (mg/dL) } \\
\hline PTD 0 & $0.6(0.4-0.9)$ & $0.6(0.4-0.9)$ & $0.6(0.4-0.9)$ & 0.972 \\
\hline PTD 1 & $0.9(0.6-1.2)$ & $0.9(0.6-1.5)$ & $0.9(0.5-1.2)$ & 0.482 \\
\hline PTD 3 & $0.9(0.6-1.4)$ & $1.7(0.9-3.5)$ & $1.0(0.7-1.5)$ & $<0.001$ \\
\hline PTD 5 & $1.3(0.8-2.1)$ & $3.1(1.5-7.2)$ & $1.3(0.8-2.3)$ & $<0.001$ \\
\hline \multicolumn{5}{|c|}{ Prothrombin time (INR) } \\
\hline PTD 0 & $1.1(1.0-1.2)$ & $1.2(1.2-1.4)$ & $1.1(1.0-1.2)$ & $<0.001$ \\
\hline PTD 1 & $1.2(1.1-1.3)$ & $1.3(1.3-1.5)$ & $1.2(1.1-1.3)$ & $<0.001$ \\
\hline PTD 3 & $1.2(1.1-1.3)$ & $1.4(1.2-1.7)$ & $1.2(1.1-1.3)$ & $<0.001$ \\
\hline PTD 5 & $1.2(1.1-1.3)$ & $1.3(1.2-1.5)$ & $1.2(1.1-1.3)$ & $<0.001$ \\
\hline \multicolumn{5}{|l|}{ Creatinine (mg/dL) } \\
\hline PTD 0 & $0.9(0.7-1.1)$ & $1.1(0.9-1.3)$ & $0.9(0.7-1.1)$ & $<0.001$ \\
\hline PTD 1 & $0.8(0.6-0.9)$ & $1.3(0.9-1.7)$ & $0.8(0.6-1.0)$ & $<0.001$ \\
\hline PTD 3 & $0.6(0.5-0.8)$ & $1.3(0.9-1.8)$ & $0.7(0.5-0.8)$ & $<0.001$ \\
\hline PTD 5 & $0.6(0.5-0.8)$ & $1.3(0.7-1.5)$ & $0.6(0.5-0.8)$ & $<0.001$ \\
\hline
\end{tabular}

Values are presented as median (interquartile range) or number (\%).

ISS, Injury Severity Score; NOM, nonoperative management; OM, operative management; INR, international normalized ratio; PTD, posttraumatic day. 


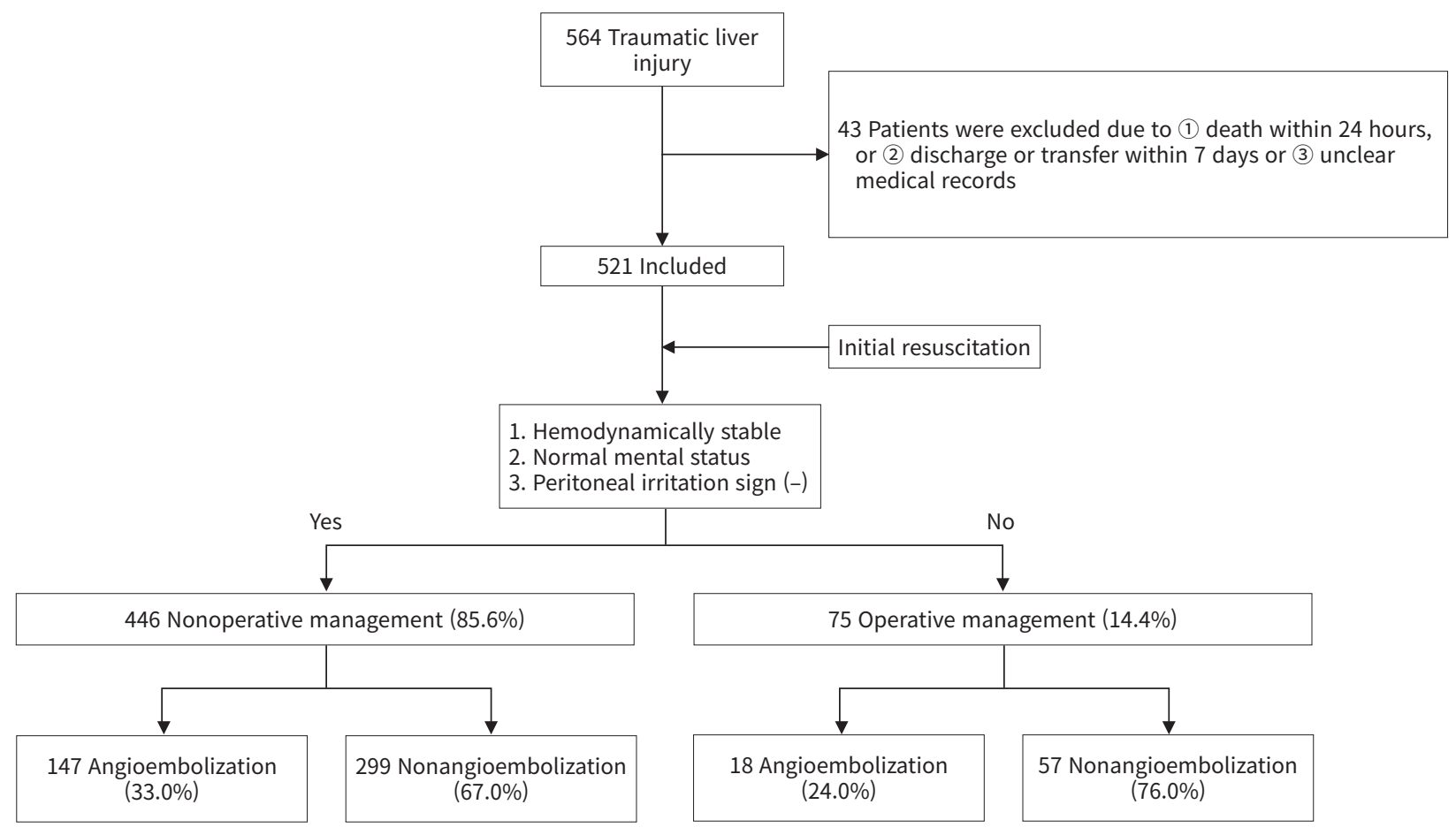

Fig. 1. Study flowchart.

Clinical characteristics of patients according to the treatment method (NOM vs. OM)

The clinical characteristics of patients according to the treatment method (NOM vs. OM) are summarized in Table S1. Median age, sex, and ISS did not significantly differ between the OM and NOM groups.

\section{Validation of the SSTLI and the cutoff point to} distinguish between low-risk and high-risk patients The application and effectiveness of the SSTLI in predicting mortality are summarized in Table 3 . The mean and standard error plots of the SSTLI are shown in Fig. 2. The effectiveness of the SSTLI for predicting mortality was significant for PTDs 0,1 , 2,3 , and $5(\mathrm{P}<0.001, \mathrm{P}<0.001, \mathrm{P}<0.001, \mathrm{P}<0.001$, and $\mathrm{P}<0.001$, respectively).

The AUCs of the SSTLI to predict mortality on PTDs $0,1,3$, and 5 were $0.736(\mathrm{P}<0.001), 0.783(\mathrm{P}<0.001), 0.830(\mathrm{P}<0.001)$, and $0.824(\mathrm{P}<0.001)$, respectively (Fig. 3). When the ability of the SSTLI to predict death was compared with that of age and ISS, the AUC of the SSTLI on PTD 0 was not statistically significantly different from that of age and ISS $(\mathrm{P}=0.081)$ (Fig. 4A). However, the AUC of the SSTLI was higher than that of age and ISS on PTDs 1,3 , and $5(\mathrm{P}=0.032, \mathrm{P}<0.001$, and $\mathrm{P}<0.001$, respectively) (Fig. 4B-D). Table 4 shows the probability of mortali- ty in our population according to the SSTLI. A very good to excellent positive correlation was observed between the probability of mortality and the SSTLI score $(\gamma=0.997, \mathrm{P}<0.001)$ (Fig. 5). Based on this result, we used a value of 5 points as the threshold to distinguish between low-risk $(<5)$ and high-risk $(\geq 5)$ patients. The sensitivity, specificity, positive predictive value, negative predictive value, and the correctly classified rate of the SSTLI with a cutoff of 5 points are shown in Table 5 .

\section{Multivariate analysis using a GEE}

Multivariate analysis using a GEE in a logistic regression model indicated that the SSTLI score was an independent predictor of mortality (odds ratio, 1.027; 95\% confidence interval, 1.0181.036; $\mathrm{P}<0.001$ ) (Table 6).

\section{DISCUSSION}

This study demonstrated the efficiency and performance of the SSTLI in predicting mortality in patients with polytraumatic liver injury. Although several scoring systems to predict the prognosis after trauma have been developed, they are not specific to traumatic liver injury. In the case of patients with trauma and chronic liver disease, chronic liver disease scoring systems such as the Child-Turcotte-Pugh system or the model for end- 
Lee et al. Validation of SSTLI

Table 3. Effectiveness of the SSTLI for predicting mortality

\begin{tabular}{lccc}
\hline SSTLI & Survivor group & Nonsurvivor group & P-value \\
\hline Overall $(\mathrm{n}=521)$ & 491 & 30 & $<0.001$ \\
PTD 0 & $2(1-3)$ & $3(2-4)$ & $<0.001$ \\
PTD 1 & $3(2-3)$ & $4(3-4.5)$ & $<0.001$ \\
PTD 3 & $3(2-4)$ & $5(4-6)$ & $<0.001$ \\
PTD 5 & $2(3.5-5)$ & $6(5-6)$ & $<0.001$ \\
NOM group $(\mathrm{n}=446)$ & 426 & 20 & $<0.001$ \\
PTD 0 & $2(1-3)$ & $3(2-4)$ & $<0.001$ \\
PTD 1 & $2(2-3)$ & $4(3-5)$ & $<0.001$ \\
PTD 3 & $3(2-3)$ & $4(4-5)$ & $5(5-6)$ \\
PTD 5 & $3(3-5)$ & 10 & 0.010 \\
OM group $(\mathrm{n}=75)$ & 65 & $3.5(3-4)$ & 0.028 \\
PTD 0 & $2(2-3)$ & $4(4-4)$ & 0.011 \\
PTD 1 & $3(2-4)$ & $6(4-7)$ & 0.006 \\
PTD 3 & $3(2-4)$ & $6(6-7)$ & \\
PTD 5 & $4(2-5)$ & & \\
\hline
\end{tabular}

Values are presented as number or median (interquartile range).

SSTLI, scoring system for traumatic liver injury; PTD, post-traumatic day; NOM, nonoperative management; OM, operative management.

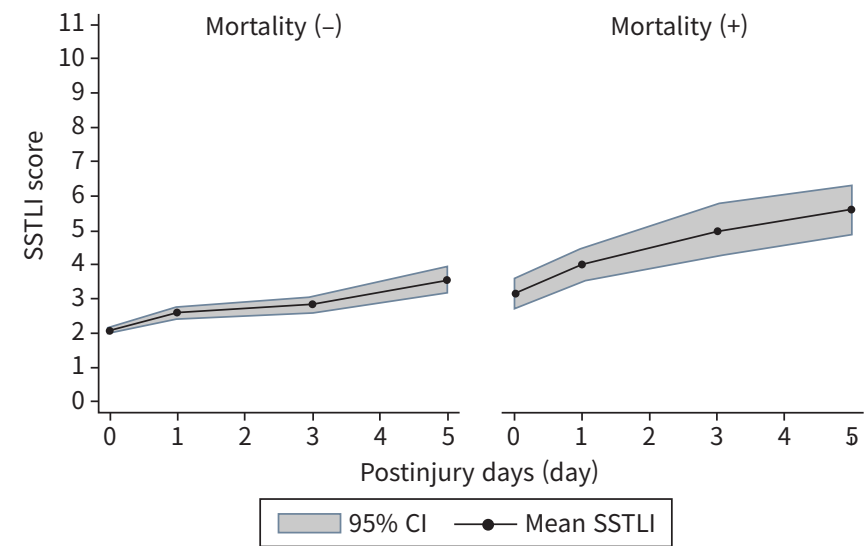

Fig. 2. Mean and standard error plots of the scoring system for traumatic liver injury (SSTLI); shaded areas represent \pm 2 standard errors. CI, confidence interval.

stage liver disease can be useful predictors of hepatic complications and the overall prognosis $[17,18]$. Hence, we developed the SSTLI in 2015 for traumatic liver injury based on the ChildPugh scoring system (Table 1) [15,19].

The SSTLI employs five clinical measures (serum total bilirubin, prothrombin time, serum creatinine, age, and the ISS) [15]. Each risk factor is assigned a point value. The discriminatory value of the SSTLI was high. In addition, the SSTLI was found to be more predictive of mortality than age or ISS alone. In this study, multivariate analysis using a GEE with a logistic regression model indicated that the SSTLI was an independent predic- tor of mortality. A cutoff of 5 points in the SSTLI was used to distinguish patients at a high-risk of mortality. With its high specificity and negative predictive value, this scoring system demonstrated the potential to rule out mortality risk. Multivariate analysis using the GEE with the logistic regression model indicated that the SSTLI was an independent predictor of mortality. These data resemble the findings of a previous study [15], and the SSTLI also predicted mortality in patients with traumatic liver injury in this study.

The SSTLI has several advantages. First, it is easy to calculate and intuitive because it involves only addition using the SSTLI table. The mortality of patients with polytrauma and liver injury can be predicted immediately. Second, the SSTLI can be calculated daily using the daily laboratory data of serum bilirubin level, prothrombin time, and creatinine level, and changes in the SSTLI can be used to predict the prognosis of patients with polytrauma and liver injury. If the SSTLI score decreases after PTD 0 , the patient's prognosis may be better. Alternatively, if the SSTLI score consistently increases or remains $>5$ points, the patient's prognosis may be worse. Third, the selected variables are indicators of poor outcomes of any injury. Therefore, we think that this model can be extended to encompass other injury types as well as liver injury.

This study was a result of follow-up with a larger group of patients over a longer period than in our previous study [15]. This study suggests that the SSTLI is useful, as it was verified to be a prognostic factor in patients with polytrauma with liver injury. 


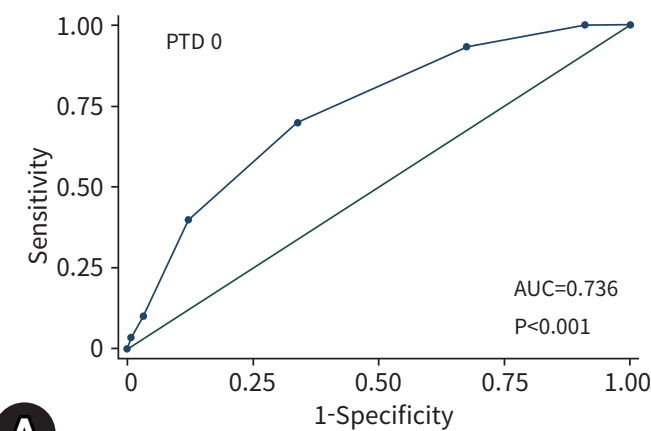

A Area under $\mathrm{ROC}$ curve $=0.7362$

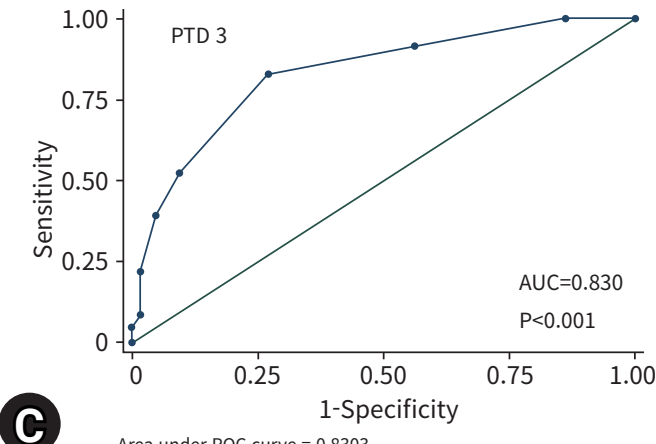

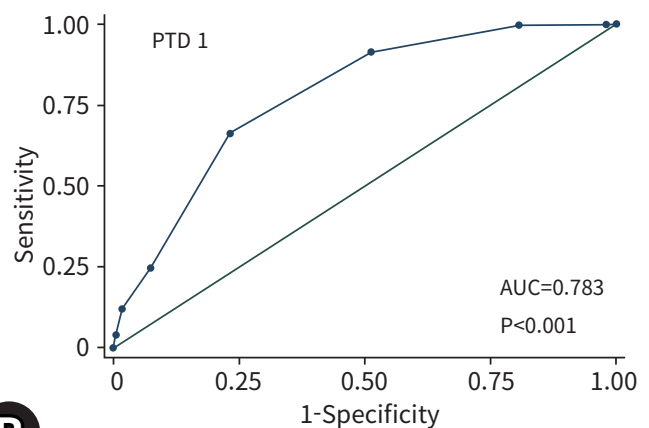

B Area under ROC curve $=0.7833$

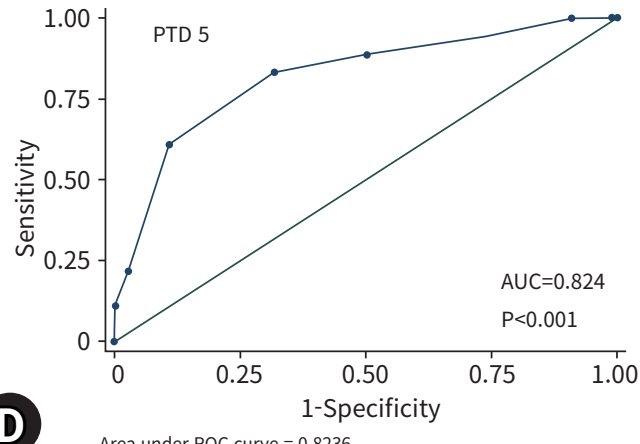

D

Fig. 3. Receiver operating characteristic (ROC) curves for the scoring system for traumatic liver injury; all areas under the ROC curves were significantly greater than that of the null area. (A) PTD 0, (B) PTD 1, (C) PTD 3, and (D) PTD 5; P<0.001. PTD, post-traumatic day; AUC, area under the curve.
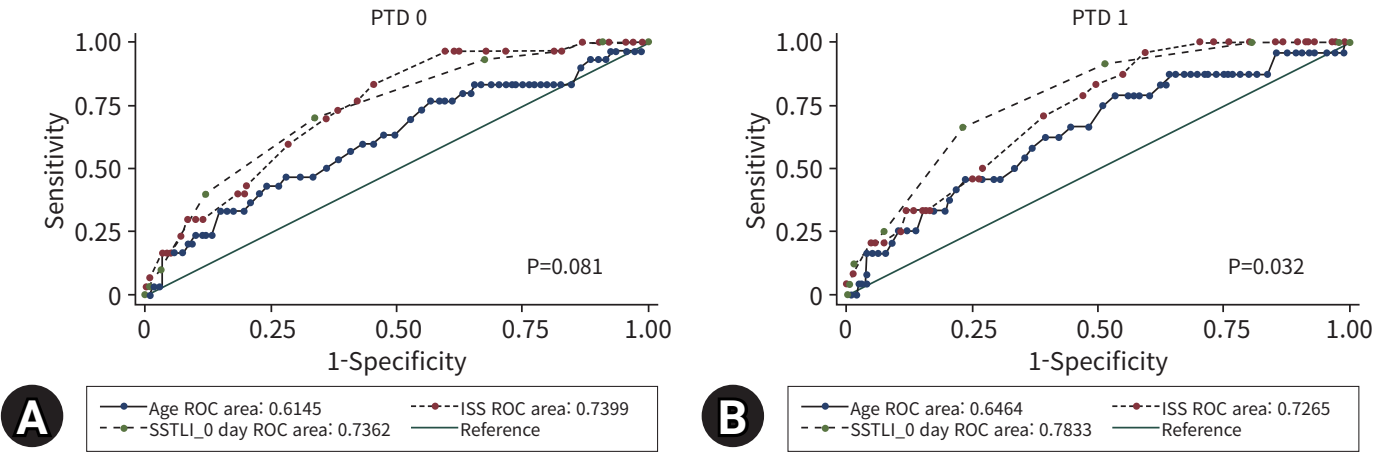

B
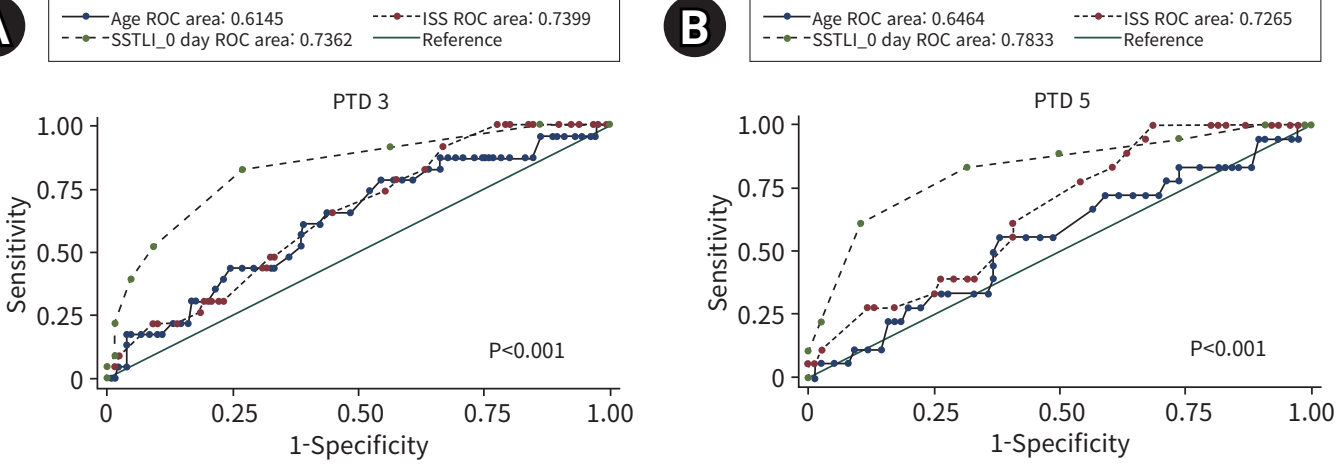

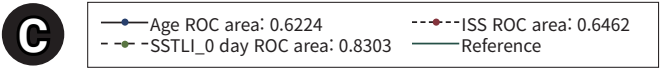
- - - SSTLI_0 day ROC area: 0.8235

Fig. 4. Receiver operating characteristic (ROC) curves for age, Injury Severity Score (ISS), and the scoring system for traumatic liver injury (SSTLI); the area under the curve of the SSTLI on (A) post-traumatic day (PTD) 0 was not statistically significantly different from that of age and ISS. However, the area under the curve of the SSTLI was greater than that of age and ISS on (B) PTD 1, (C) PTD 3, and (D) PTD 5. 
Table 4. Risk of mortality in patients with polytrauma and liver injury according to the SSTLI

\begin{tabular}{lccccc}
\hline \multirow{2}{*}{ Score } & \multicolumn{5}{c}{ Probability of mortality (\%) } \\
\cline { 2 - 5 } & PTD 0 & PTD 1 & PTD 3 & PTD 5 & \\
\hline 0 & 0 & 0 & - & 0 & 0 \\
1 & 1.7 & 0 & 0 & 0 & 0.4 \\
2 & 4.1 & 2.4 & 4.9 & 7.1 & 4.6 \\
3 & 7.8 & 7.1 & 5.0 & 5.3 & 6.3 \\
4 & 17.0 & 18.5 & 23.3 & 6.7 & 16.4 \\
5 & 15.4 & 14.8 & 33.3 & 20.0 & 20.9 \\
6 & 20.0 & 40.0 & 50.0 & 53.9 & 41.0 \\
7 & - & 50.0 & 100.0 & 50.0 & 66.7 \\
8 & - & - & 33.3 & 100.0 & 66.7 \\
9 & - & - & 100.0 & - & 100.0 \\
\hline
\end{tabular}

SSTLI, scoring system for traumatic liver injury; PTD, post-traumatic day.

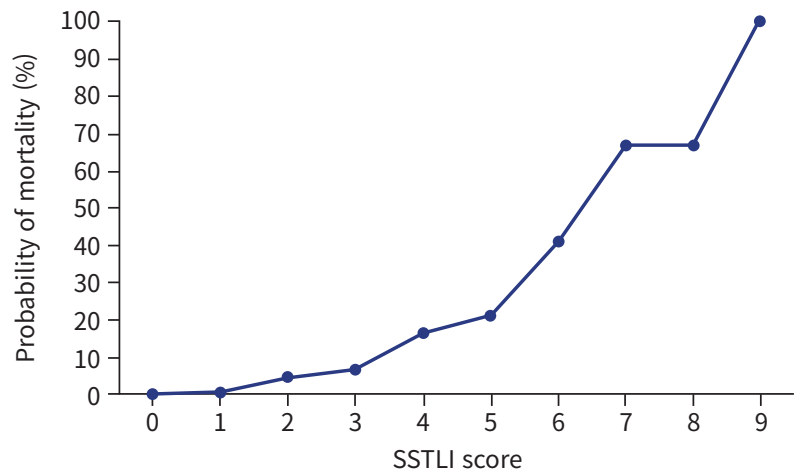

Fig. 5. Correlation between the probability of mortality in patients with polytrauma and liver injury and the scoring system for traumatic liver injury (SSTI; Spearman rho=0.997, P<0.001; SSTLI 0-SSTLI 5).

Table 5. Sensitivity, specificity, positive predictive value, and negative predictive value of the SSTLI with a cutoff of 5 points

\begin{tabular}{lcccccc}
\hline PTD & Sensitivity (\%) & Specificity (\%) & PPV (\%) & NPV (\%) & Correctly classified (\%) & Prevalence (\%) \\
\hline 0 & 10.0 & 96.9 & 16.7 & 94.6 & 91.9 & 5.8 \\
1 & 25.0 & 92.8 & 23.1 & 93.5 & 87.4 & 7.9 \\
3 & 52.2 & 90.8 & 50.0 & 91.5 & 85.0 & 15.0 \\
5 & 83.3 & 68.4 & 38.5 & 94.5 & 71.3 & 19.1 \\
\hline
\end{tabular}

SSTLI, scoring system for traumatic liver injury; PTD, post-traumatic day; PPV, positive predictive value; NPV, negative predictive value.

Table 6. Multivariate analysis using a generalized estimating equation extension of the logistic regression model for patients with traumatic liver injury

\begin{tabular}{lcccc}
\hline Variable & Z-score & Estimate (SE) & OR (95\% CI) & P-value \\
\hline SSTLI & 5.84 & $0.027(0.005)$ & $1.027(1.018-1.036)$ & $<0.001$ \\
\hline
\end{tabular}

SE, standard error; OR, odds ratio; CI, confidence interval; SSTLI, scoring system for traumatic liver injury.

This study has several limitations. First, there are many other variables that could have been considered, but were not included in our model (e.g., serum levels of base deficit and lactic acid increase in the case of sepsis, multiple organ dysfunction, and shock) [20-24]. Second, age and ISS, which are the major factors we used in the scoring system, are themselves major factors already used to predict the prognosis of trauma patients. In addition, the question remains of whether serum total bilirubin, prothrombin time, and creatinine are specific factors related to liver injury in trauma patients. In the future, a comparative study between patients without liver damage and those with liver injury using this scoring system would be helpful. Finally, this study included patients from a single center and also was a nonrandomized, retrospective analysis. Our study population might be specific to a region, which might limit the generaliz- ability of our findings. External validation would be required to confirm the generalizability of the SSTLI. Additional prospective, randomized, controlled trials with larger sample sizes are necessary to confirm the validity of the SSTLI.

In conclusions, we investigated prognostic factors in patients with traumatic liver injury and reaffirmed the validity of the SSTLI to predict mortality. An SSTLI score of 5 or higher indicated a high-risk of post-traumatic mortality. Our study suggests that the SSTLI could be used to predict mortality in patients with traumatic liver injury. Patients who score 5 or higher on the SSTLI should be monitored particularly carefully.

\section{SUPPLEMENTARY MATERIALS}

Fig. S1. Receiver operating characteristic (ROC) curves for (A) age and (B) Injury Severity Score (ISS); the area under the curve (AUC) for age and ISS were significantly greater than the null area $(\mathrm{P}<0.01)$.

Table S1. Clinical characteristics of patients with traumatic liver injury according to the treatment method

Supplementary materials are available from: https://oi.org/ 10.20408/jti.2021.0009. 


\section{NOTES}

\section{Ethical statement}

This study was approved by the Institutional Review Board of the Pusan National University Hospital, Busan (No. H-2012-022098).

\section{Conflicts of interest}

The authors have no conflicts of interest to declare.

\section{Funding}

This work was supported by a clinical research grant from $\mathrm{Pu}$ san National University Hospital in 2021.

\section{Author contributions}

Conceptualization: RDY, KHH; Data curation: RDY; Formal analysis: KHH; Investigation: RDY; Methodology: LGH, JCH, KHH; Project administration: RDY, KHH; Resources: LGH, KHH; Supervision: KJH, YSR; Validation: KHH, KJH; Visualization: JCH; Writing-original draft: LGH; Writing-review \& editing: KHH, PCY, YSR.

All authors read and approved the final copy of the manuscript.

\section{REFERENCES}

1. Arumugam S, Al-Hassani A, El-Menyar A, et al. Frequency, causes and pattern of abdominal trauma: a 4-year descriptive analysis. J Emerg Trauma Shock 2015;8:193-8.

2. Smith J, Caldwell E, D'Amours S, Jalaludin B, Sugrue M. Abdominal trauma: a disease in evolution. ANZ J Surg 2005; 75:790-4.

3. Costa G, Tierno SM, Tomassini F, et al. The epidemiology and clinical evaluation of abdominal trauma. An analysis of a multidisciplinary trauma registry. Ann Ital Chir 2010;81: 95-102.

4. She WH, Cheung TT, Dai WC, et al. Outcome analysis of management of liver trauma: a 10-year experience at a trauma center. World J Hepatol 2016;8:644-8.

5. Brillantino A, Iacobellis F, Festa P, et al. Non-operative management of blunt liver trauma: safety, efficacy and complications of a standardized treatment protocol. Bull Emerg Trauma 2019;7:49-54.

6. Velmahos GC, Toutouzas K, Radin R, et al. High success with nonoperative management of blunt hepatic trauma: the liver is a sturdy organ. Arch Surg 2003;138:475-80.

7. Virdis F, Reccia I, Di Saverio S, et al. Clinical outcomes of pri- mary arterial embolization in severe hepatic trauma: a systematic review. Diagn Interv Imaging 2019;100:65-75.

8. Bertens KA, Vogt KN, Hernandez-Alejandro R, Gray DK. Non-operative management of blunt hepatic trauma: does angioembolization have a major impact? Eur J Trauma Emerg Surg 2015;41:81-6.

9. Stassen NA, Bhullar I, Cheng JD, et al. Nonoperative management of blunt hepatic injury: an Eastern Association for the Surgery of Trauma practice management guideline. J Trauma Acute Care Surg 2012;73(5 Suppl 4):S288-93.

10. Cimbanassi S, Chiara O, Leppaniemi A, et al. Nonoperative management of abdominal solid-organ injuries following blunt trauma in adults: results from an International Consensus Conference. J Trauma Acute Care Surg 2018;84:517-31.

11. Knaus WA, Draper EA, Wagner DP, Zimmerman JE. APACHE II: a severity of disease classification system. Crit Care Med 1985;13:818-29.

12. Baker SP, O’Neill B, Haddon W Jr, Long WB. The Injury Severity Score: a method for describing patients with multiple injuries and evaluating emergency care. J Trauma 1974;14: 187-96.

13. Champion HR, Sacco WJ, Copes WS, Gann DS, Gennarelli TA, Flanagan ME. A revision of the Trauma Score. J Trauma 1989;29:623-9.

14. Kimura A, Chadbunchachai W, Nakahara S. Modification of the Trauma and Injury Severity Score (TRISS) method provides better survival prediction in Asian blunt trauma victims. World J Surg 2012;36:813-8.

15. Kim HH, Kim JH, Park CY, Cho HM. Scoring system for traumatic liver injury (SSTLI) in polytraumatic patients: a predictor of mortality. Eur J Trauma Emerg Surg 2015;41: 375-85.

16. Zeger SL, Liang KY, Albert PS. Models for longitudinal data: a generalized estimating equation approach. Biometrics 1988; 44:1049-60.

17. Corneille MG, Nicholson S, Richa J, et al. Liver dysfunction by model for end-stage liver disease score improves mortality prediction in injured patients with cirrhosis. J Trauma 2011; 71:6-11.

18. Talving P, Lustenberger T, Okoye OT, et al. The impact of liver cirrhosis on outcomes in trauma patients: a prospective study. J Trauma Acute Care Surg 2013;75:699-703.

19. Child CG. The liver and portal hypertension. Major problems in clinical surgery. Vol. 1. Philadelphia: Saunders; 1964.

20. Plessier A, Denninger MH, Consigny Y, et al. Coagulation disorders in patients with cirrhosis and severe sepsis. Liver 
Int 2003;23:440-8.

21. Moseley RH. Sepsis and cholestasis. Clin Liver Dis 2004;8:8394.

22. Ferreira AM, Sakr Y. Organ dysfunction: general approach, epidemiology, and organ failure scores. Semin Respir Crit Care Med 2011;32:543-51.

23. Kelley KC, Dammann K, Alers A, Zanders TB, Bendas C, Stawicki SP. Resuscitation endpoints in traumatic shock: a fo- cused review with emphasis on point-of-care approaches. In: Stawicki SP, Swaroop M, editors. Clinical management of shock: the science and art of physiological restoration. London: Intech Open; 2020.

24. Di Saverio S, Catena F, Filicori F, et al. Predictive factors of morbidity and mortality in grade IV and V liver trauma undergoing perihepatic packing: single institution 14 years experience at European trauma centre. Injury 2012;43:1347-54. 